\title{
Gentrification and Densification as Determining Factors of Orientation and Quality of Neighbors Relations in Indonesia
}

\section{(A Hypothetical Study)}

\author{
Ichlas Nanang Afandi \\ Department of Psychology \\ Hasanuddin University \\ Indonesia \\ ichlasafandi@yahoo.co.id
}

\begin{abstract}
In a society with highly diverse psycho-social attributes (ethnic group, religion, social-economic status, etc.) such as Indonesia, the positive orientation and quality of relations between neighbors are necessary to prevent things which can harm the harmony of the society. Today, by hypothetical, the orientation and quality of relations between neighbors in Indonesia have changed. In the past, the orientation of relations between neighbors was oriented toward community benefit which is associated with positive quality of relations between neighbors. Now, the orientation of relations between neighbors was oriented toward material benefit which is associated with negative quality of relations between neighbors. Many studies state that quality of relation is one of the determinants of individual well-being. Many factors which influence the orientation and quality of relations between neighbors in Indonesia, but now gentrification (the entry of the rich to the neighborhoods of the poor) and densification (opening new settlement areas around old) are two major factors which had a significant role in the condition, especially in large cities with dynamic and massive socio-economic movements, such as Jakarta, Surabaya, Makassar, Bandung, Medan, and Yogyakarta. This paper is a hypothetical study on the influence of gentrification and densification on the orientation and quality of relations between neighbors in Indonesia.
\end{abstract}

Keywords-Gentrification, Densification, Relation, Neighbors, and Well-Being

\section{INTRODUCTION}

In a country with high diverse psycho-social attributes such as Indonesia (religion, ethnicity, socioeconomic status, etc.), one of the things that needs more attention is the issue of relations between neighbors. The orientation and quality of relations between neighbors determine the extent to which harmony in a community can be maintained, which ultimately determines how much potential horizontal conflict can occur (macro level) and individual psychological wellbeing (micro level). The more complex the psycho-social attributes of society in a country, will affect complexity relations between neighbors. So it can be imagined how the complex dynamics of relations between neighbors that occur in a country with a very varied community attributes such as Indonesia. More complex because of modernity as a result of social change with various variations of demands in it also influences how people define relationships with people around him (neighbors).

In addition to being characterized by a variety of psychosocial attributes, Indonesia is also known as an interdependent collectivist state with a pattern of neighboring relations based on community orientation. In a country with interdependent collectivistic culture, the relationships between the constructed individuals are more socially beneficial [1]. Collectivist societies more closely linked between individuals and their opinions and beliefs focus on a group or community [2]. Similarly, community-based relationships are relationships established by individuals based on norms and social orientations (intangible transactions of matter) and focus on how individuals are responsible for other individuals [3]. The condition as a model of communal sharing that is a relationship model that is based on the conception of an equal and undifferentiated group, meaning that individuals within a group or society do not differentiate certain identity from each other and focus on togetherness [3].

However, neighbors relations oriented to the benefit of the community seem to have been abandoned by the people of Indonesia. There is a symptom of a shift in social relations becoming more exchange-oriented based on material gains, as in individualistic-independent countries. In some residential areas in Indonesia, especially in big cities such as Jakarta, Surabaya, Makassar, Medan, Yogyakarta, or Bandung, there are symptoms that characterize the shift in question, such as not knowing each other well, low involvement in society, ignorant (apathetic) for what happens in the neighbors environment, until attitudes and behaviors that are based solely on the consideration of economic benefits and losses. In the context of neighbors' relations, the last sentence is probably the most alarming symptom. 


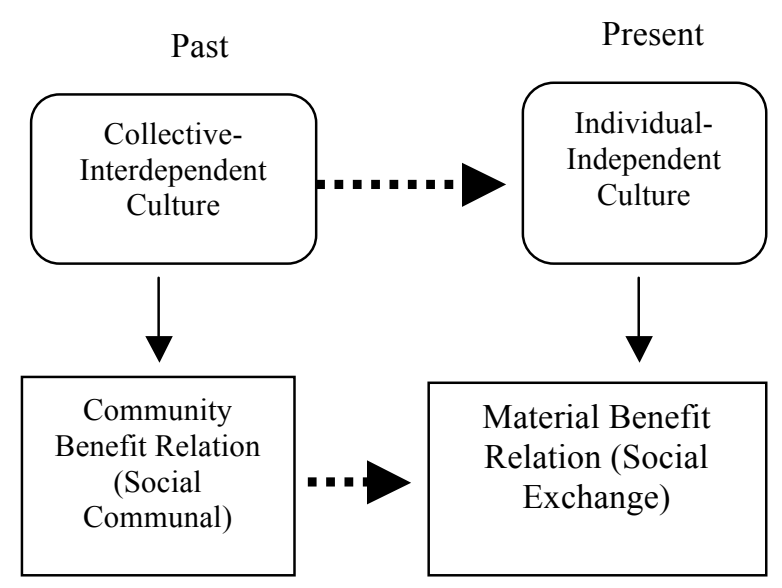

Figure 1. Hypothesis of shifting relations between neighbors in Indonesia.

These conditions are important to note further, given the orientation and quality of social relationships determine many aspects of life, from the macro aspect (harmony and potential horizontal conflict) to the micro aspect (well-being). Many of research has found a relationship between the orientation and quality of relationships with harmony and potential conflicts in society, and well-being in individuals. Individuals who have quality social relationships, such as a harmonious marriage, having close friends, being in a friendly atmosphere, and a supportive coworker, it's harder to feel sadness, loneliness, low self-esteem, and eating and sleeping problems [4].

There are many factors that determine the orientation and quality between neighbors. Several factors that determine the orientation and quality of relations between neighbors, namely: environmental conditions (topographic aspects), physical distance between neighbors, the availability of transportation, types of existing buildings, infrastructure (roads), demographic, SES, ethnicity, population movement), the existence and quality of public services, patterns of interaction with friends and family, community (formal and informal), regional sentiments, and political conditions [5]. However, at present, there are two main and actual factors that can be explained as to why there is a shift in the orientation of relations between neighbors in Indonesia, especially in big cities with massive socio-economic movement, Gentrification (the activity of the entry of the rich to the poor settlements) and Densification (new settlement land clearing activity around the old settlement). Social mixing, gentrification, and densification are actual factors that determine the pattern and quality of relations between neighbors [6].

Like the big cities of the world, the phenomenon of gentrification and densification is also prevalent in big cities in Indonesia. Gentrification (also densification) is currently a global phenomenon that occurs almost in every country in the world [7]. Such rapid socio-economic movements, impacting massive population movements, ultimately lead to the demand for a modern and viable settlement amidst the denser neighborhoods. On the one hand, gentrification and densification appear to have a positive economic impact on citizens. But on the other hand, the phenomenon affects the social structure of society, one of which is the shift of orientation and patterns of relations between neighbors, which was once oriented community into a material orientation.

This paper is a hypothetical study of how gentrification and densification affect the shift in the orientation and quality of relations between neighbors in Indonesia.

\section{GENTRIFICATION AND DENSIFICATION}

In terminology, gentrification and densification are two different things. However, in the context of the discussion surrounding the use of land for resettlement, the two terms cannot be separated from each other. According to the Urban Geography dictionary, gentrification is the process of clearing land in urban areas that were initially neglected, with the aim that residents previously living in the area obtain easy access to jobs and public services (previously inaccessible) [6]. Gentrification as the entry activity of the rich (later called gentrifies) to build houses in poor settlements (called locals). Meanwhile, densification is defined as the activity of opening new settlements in old residential areas (pre-existing ones) [6].

Gentrification and densification have a positive and negative impact on people's lives. The positive impact is more on the economic aspects (increasing local economic conditions), while the negative impact on social aspects (social relations). These conditions make the phenomenon of gentrification and densification debated by some parties [7]. Gentrification (also densification) has become a controversial topic of discussion. The existing debate develops in the discussion of whether gentrification and densification should be supported (weighing the positive impact it incurs), or opposed (weighing the negative impact it also generates), or it should be controlled for its viability [8].

\section{NEIGHBORS RELATION}

\section{A. Defenition}

Relations between neighbors as the actual and observed interactions that occur between neighbors [9]. Then, relations between neighbors as social relations that occur between individuals living in adjacent locations [10]. Furthermore, relationship between neighbors as a social community that lives close together and in it happens friendship among members [11]. Relations between neighbors as co-operative conditions that occur between members in a social community (neighboring communities) [12]. Relationships between neighbors as interactions between individuals living in close proximity in a particular area that has implications for the formation of social communities [13]. Relations between neighbors as a formal and informal interaction that occurs between members of a population within a given region [14].

A much more complex definition is relationships between neighbors as spontaneous interpersonal interactions, 
resulting in a sense of attachment, a structure and a relatively similar purpose, friendship, jokes, and a container for expressing self [15]. Then, relations among neighbors are generally defined as spatial units in which a "face-to-face" social interaction takes place which then generates the values and rules mutually agreed upon by each of its members [16]. Furthermore, relations between neighbors are a condition that is marked by the contact, interaction, meeting, or closeness between one person with another person, which is more common in informal settings [17]. It is said to be more complex because the proposed definition involves the dimensions involved in the relations between neighbors (although the dimensions of relations between neighbors will be described separately).

\section{B. Factors Determining the Orientation and Quality of Relationship between Neighbors}

There are many factors that determine the quality, pattern, and dynamics of relations between neighbors. Two factors that determine the quality of relations between neighbors are: (1) Environmental condition factors, called natural factors, and (2) Social conditions that occur, called social factors, such as characteristics the individuals involved, the demographic conditions in which the relationships take place, the interaction system that occurs, how the sharing patterns occur, and the symbols used [14]. Furthermore, relations between neighbors are determined by local economic factors, prevailing public policies, as well as changes in sociocultural conditions [18].

Relations between neighbors are assessed so it is necessary to consider several determinants: environmental conditions (topography aspect), physical distance between neighbors, availability of transportation, types of existing buildings, infrastructure (roads), demographic aspects (age of population , SES, ethnicity, population movement), the existence and quality of public services, patterns of interaction with friends and family, community (formal and informal), regional sentiments, and political conditions [5]. Social mixing, gentrification (and the entry of rich people into the poor neighborhood), and densification (the recent settlement activity of old settlements) are actual factors that determine the pattern and quality of relations between neighbors [6].

\section{GENTRIFICATION AND DENSIFICATION, NEIGHBORS RELATION, AND WELL-BEING}

One effect of gentrification and densification is the emergence of social issues, one of them on the orientation and quality of neighbors' relation. Gentrification and densification can be the trigger factor of conflict between citizens, namely between the rich (gentrifies) with the poor (local people) [10]. In addition, gentrification and densification become factors that further widen the distance between the rich (gentrifies) with the poor (local people). One of the social impacts of gentrification and densification is the reduced intensity of social interaction (social relations) between citizens (between neighbors) [6].

Meanwhile, several studies have found a close relationship between the qualities of social relationships with the health (physical and psychological) of individuals. Many research facts have found the role of social relationships to our physical and psychological health [19]. One of them, that individuals who have quality social relationships, such as a harmonious marriage, have close friends, are in a friendly neighborhood, and supportive colleagues will find it harder to feel sad, loneliness, low self-esteem, and eating and sleeping problems [4]. Well established social networks will form more stable emotional states, more orderly behavior, and an opportunity to be more emotionally attached to others. Such conditions will minimize the onset of illness (physical), conflict, stress, feelings of loss and loneliness [19].

\section{CONCLUSION}

Gentrification and densification is a social reality. Some parties call it necessary because it has a positive impact on the increasing level of economic community. However, on the other hand, some circles are also opposed because of the negative impact on social aspects it produces. In the Indonesian context, especially in big cities, gentrification and densification are also underway. Positive and negative impacts have also been felt, although negative impacts are much more visible. Hypothetically, there has been a shift in orientation and the quality of social relations (between neighbors) caused by gentrification and densification.

This paper is a hypothetical study, so it is open space for anyone to criticize it. For that, it is desirable for other researchers to follow up this study in the form of field research, so that the existing hypothesis can be cut into a thesis which of course has more scientific strength and smaller space to be criticized.

\section{REFERENCES}

[1] L. Sundarajan, Understanding Emotions in Chinese Culture : Thinking Thorugh Psychology. Springer International Publishing Switzerland. 2015

[2] P.A. Gambrel \& R. Cianci, Maslow's Hierarchy of Needs: Does It Apply In Collectivist Culture. The Journal of Applied Management and Entrepreneurship, 8 (2), 143. 2003

[3] A.P. Fiske, The Four Elementary Forms of Sociality: Framework for a Unified Theory of Social Relations. American Psychological Association, 99(4), 689-723. 1992

[4] J.F. Helliwell \& R.D. Putnam, The Social Context of Well-Being. The Royal Society Journal. 2004.

[5] G. Galster, On The Nature of Neighbourood. Urban Studies, 38, (12), 2111-2124. 2001

[6] L, Cheshire., R, Fitzgerald, R., A, Clarke, \& S, Raymond, Neighbourly Problems in Neighbourhood Context: Understanding How Neighbourhoods Influence the Prevalence of Neighbourly Problems and Complaints. Paper Presented to the session 'How much do Urban Neighbourhoods Matter in a Networked Globalised Space?. RC21 Conference. Berlin, Germany. 2013

[7] R, Atkinson, \& G, Bridge, Gentrification in Global Context : The New Urban of Colonalism. Abingdon : Routledge, Taylor \& Francis Group. 2005

[8] M, Bernt, \& A, Holm, A, 'Is it, or Is Not? The Conceptualisation of Gentrification and Displacement and its Political Implications in The Case of Berlin- Prenzlauer Berg', City 13, pp. 312-324. 2009

[9] H, Rounavaara, \& A, Kouvo, Neighbour Relation in Contemporary Society. A Survey of Ideas and a Blueprint for a Framework for 
Investigation. A Paper Presented in The ISA Housing Assets, Housing People Conference in Glasgow, 1-4th September 2009.

[10] A, Buonfino \& P, Hilder, Neighbouring in Contemporary Britain. Joseph Rowntree Foundation. 2006

[11] D, Harper, Neighbourhood : Online Etimology Dictionary. Retrived from www.etimomline.com, on march 16th 2015.

[12] I, Scridon, Neighbourhood Relationship Between German Ethnic Groups from Romania. Case Study : The Zipsers from Viseu de Sus, Maramures County. Journal of Settlements and Spatial Planing, 3, (1 ), $51-56.2012$

[13] M.G, Berk, The Concept of Neighbourhood in Contemporary Residental Environments : An Investigation of Occupant's Perception. Munich Personal RePEc Archive. 2005.

[14] K.P, Schwirian, Models of Neighbourhood Change. Annual Review of Sociology, 9, 83-102. 1983
[15] E.A, Greenfield \& L, Reyes, Continuity and Change in Relationships with Neighbors: Implications for Psychological Well-Being in Middle and Later Life. Journals of Gerontology, Series B, Psychological Sciences and Social Sciences. 2014

[16] A, Schuck \& D, Rosenbuam, Promoting Safe and Healthy Neighborhoods: What Research Tells Us about Intervention. The Aspen Institute. 2006

[17] G, Mollenhorst., B, Volker \& Schutjens, Neighbour Relations in The Netherlands : A Decade of Evidence. Royal Dutch Geographical Society KNAG. 2009

[18] R, Johnston., M, Poulsen \& J, Forrest, The Geography of Ethnic Residential Segregation: A Comparative Study of Five Countries. Annals of the Association of American Geographers, 97,( 4), 713-738. 2007

[19] S, Cohen, Social Relationship and Health. American Psychologist Journal. 2004. 